\title{
Structural insights into the phlorotannin binding of EHEP and akuBGL for producing biofuel from brown algae
}

\author{
Xiaomei Sun ${ }^{1}$, Yuxin Ye1, Naofumi Sakurai ${ }^{1}$, Koji Kato', Jian Yu ${ }^{1}$, Keizo Yuasa ${ }^{2}$, Akihiko Tsuji ${ }^{2}$, Min Yao ${ }^{1}$ \\ ${ }^{1}$ Faculty of Advanced Life Science, Hokkaido University; \\ ${ }^{2}$ Graduate School of Bioscience and Bioindustry, Tokushima University \\ sun@sci.hokudai.ac.jp
}

The world is meeting the challenge of the energy crisis, which prompted searching for renewable energy resource. Brown algae are ideal feedstocks for producing biofuels, the promising renewable resource. The sea hare Aplysia kurodai is an excellent model for investigating the biofuel production process. It consumes brown algae as a staple food to release a large amount of glucose from laminarin-depolymerization by the $\beta$-glucosidase in its digestive fluid (akuBGL). However, brown algae produced abundant secondary metabolite as a defense against the herbivores, such as phlorotannin. Phlorotannin inhibits akuBGL activity, which causes a problem in biofuel production from brown algae. Interestingly, Eisenia hydrolysis enhancing protein (EHEP) existed in the digestive fluid of Aplysia kurodai and, was found to protect akuBGL from phlorotannin-inhibition by binding with phlorotannin and precipitating ${ }^{1}$. How EHEP bind to phlorotannin, why it can protect $a k u$ BGL from inhibition both are unknown.

In this study, we obtained EHEP and $a k u$ BGL from digestive fluid of A. kurodai. We determined the structures of EHEP in apo form and complex with an analogue of the phlorotannin, tannic acid by native-SAD method ${ }^{2}$. The structures reveal that EHEP consisted of three chitin-binding domains linked by two long loops (Fig.1) and tannic acid bound at the center of EHEP. Furthermore, we determined the structure of $a k u \mathrm{BGL}$ and showed it comprised of two GH1 (glycoside hydrolysis family 1) domains linked by the loop (Fig.2). Additionally, the docking analysis of tannic acid with akuBGL was performed, revealing the tannic acid occupies the active pocket of $a k u$ BGL. Collectively, we proposed the mechanism of EHEP that protects $a k u$ BGL from inhibition.

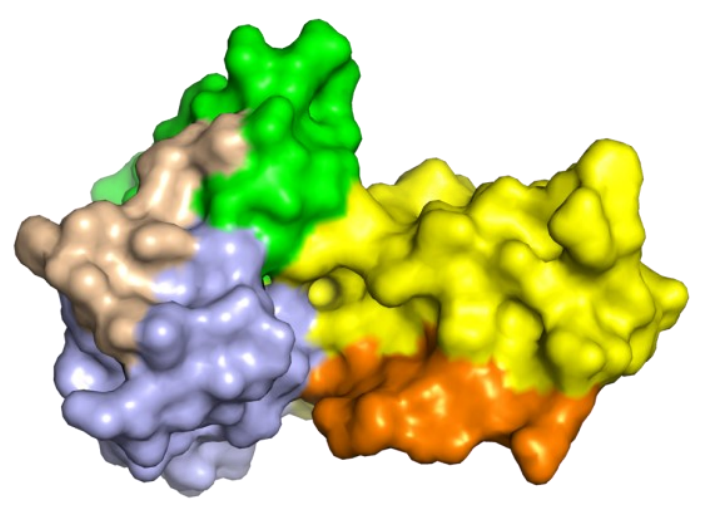

Figure 1. Complex structure of EHEP-tannic acid

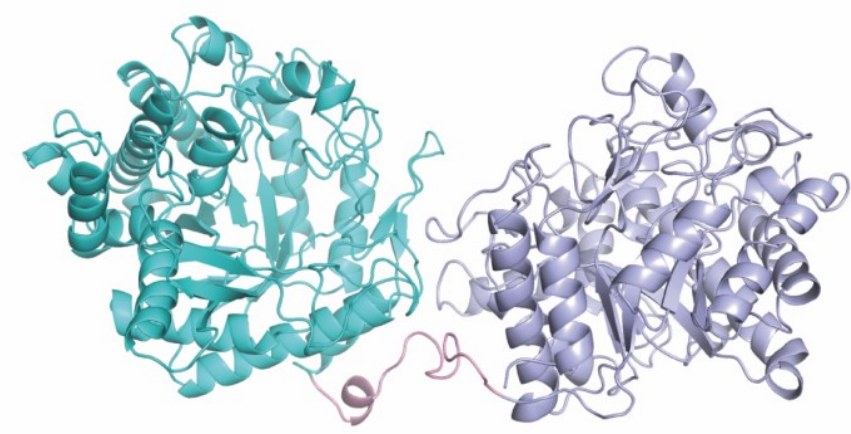

Figure 2. $a k u \mathrm{BGL}$ structure

[1] Tsuji, A., Kuwamura, S., Shirai, A. \& Yuasa, K. (2017). PLoS One 12, e0170669.

[2] Sun, X., Ye, Y., Sakurai, N., Kato, K., Yuasa, K., Tsuji, A. \& Yao, M. (2020). Acta Crystallographica Section F-Structural Biology Communications 76, 20-24

Keywords: EHEP; akuBGL; biofuel; phlorotannin binding 